\title{
Composites and Nanocomposites Based on Renewable and Sustainable Materials
}

\author{
Matheus Poletto, ${ }^{1}$ Heitor Luiz Ornaghi Júnior, ${ }^{2}$ P. M. Visakh, ${ }^{3}$ and Yoshihiko Arao ${ }^{4}$ \\ ${ }^{1}$ Centro de Ciências Exatas, da Natureza e Tecnologia, Universidade de Caxias do Sul, 95700-000 Caxias do Sul, RS, Brazil \\ ${ }^{2}$ Programa de Pós-Graduação em Engenharia e Ciências dos Materiais (PGMAT), Universidade de Caxias do Sul, \\ 95070-560 Caxias do Sul, RS, Brazil \\ ${ }^{3}$ Tomsk Polytechnic University, Tomsk 634050, Russia \\ ${ }^{4}$ Tokyo Institute of Technology, Tokyo 152-8550, Japan
}

Correspondence should be addressed to Matheus Poletto; matheus.poletto@hotmail.com

Received 11 May 2016; Accepted 12 May 2016

Copyright (C) 2016 Matheus Poletto et al. This is an open access article distributed under the Creative Commons Attribution License, which permits unrestricted use, distribution, and reproduction in any medium, provided the original work is properly cited.

Renewable and sustainable materials are of great concern in scientific research and industry. These materials include biomass as industrial and agricultural residues and energy crops. The utilization of such materials as reinforcement or to improve specific properties in composite or nanocomposites materials is an excellent alternative. Among the advantages which can be cited are the strong ecological appeal, low cost, and higher specific mechanical properties.

Topics include the effect of the chemical treatment on the physicomechanical properties of commercial polypropylene, preparation of nanofibers with renewable polymers and their application in wound dressing, use of waste clay from boron production as landfill liner, use of wood fillers in thermoplastic matrix, and thermal insulation of paper cups.

The effects of reinforcement of the treated and untreated banana bark fiber on the physicomechanical properties of isotactic polypropylene were verified, and the water absorption, mechanical properties, and hardness, among other properties, were improved up to treatment. Another study includes the effect of fiber content on mechanical and morphological properties of woven kenaf reinforced PVB film produced using a hot press technique. In this study, the authors produced six different types of laminated composites and their mechanical properties were compared. The authors constated improvement of the mechanical characteristics up to $40 \%$ kenaf content ratio.

In a review study regarding preparation of nanofibers with renewable polymers and their application in wound dressing, the authors constated that the preparation methods as well as the components influenced their overall properties.

Wood fibers also were used as reinforcement in HDPE composites. The rheological and the thermomechanical properties of composites vary according to wood filler content and temperature.

A facile approach to evaluate the thermal insulation performance of paper cups was realized by establishing a novel evaluation that includes a characterization parameter and a measurement apparatus, where the value of the temperature factor is proportional to the thickness of paper samples.

Dynamic analysis of the melt conveying process in a novel extruder as studied and the researcher concluded that the polymer melt conveying process in the vane extruder is completely different from the process in conventional screw extruders; the pressure within the chamber is influenced by the location and volume within the chamber and the dynamics of melt conveyance in a vane chamber depend mostly on pressure to overcome viscous forces.

The effect of the chitosan on isothermal crystallization, thermal decomposition, and melt index of PLLA polymer was studied. The authors concluded that chitosan acts as a nucleating agent for PLLA, affecting the initial degradation temperature and leading to higher values of melt flow index up to $3 \%$ of chitosan.

Finally, boron production waste clay was used to develop a geosynthetic barrier that can be used as an innovative impermeable liner on solid waste landfills. In this study, 
waste clay generated during boron derivatives production was mixed with bitumen, bentonite, and waste tire and the mixtures were used to cover up reinforcement materials (nonwoven polyester and glass fleece). The samples were compared to commercial geomembranes and three samples were used to apply hydraulic conductivity test. Microscopy and mechanical and conductivity tests were performed and satisfactory results were found. The study allows the production of lower cost geomembranes while leading up to utilizing waste clay of 900,000 tons/year generated at boron enterprises in Turkey.

Matheus Poletto

Heitor Luiz Ornaghi Júnior

P. M. Visakh

Yoshihiko Arao 

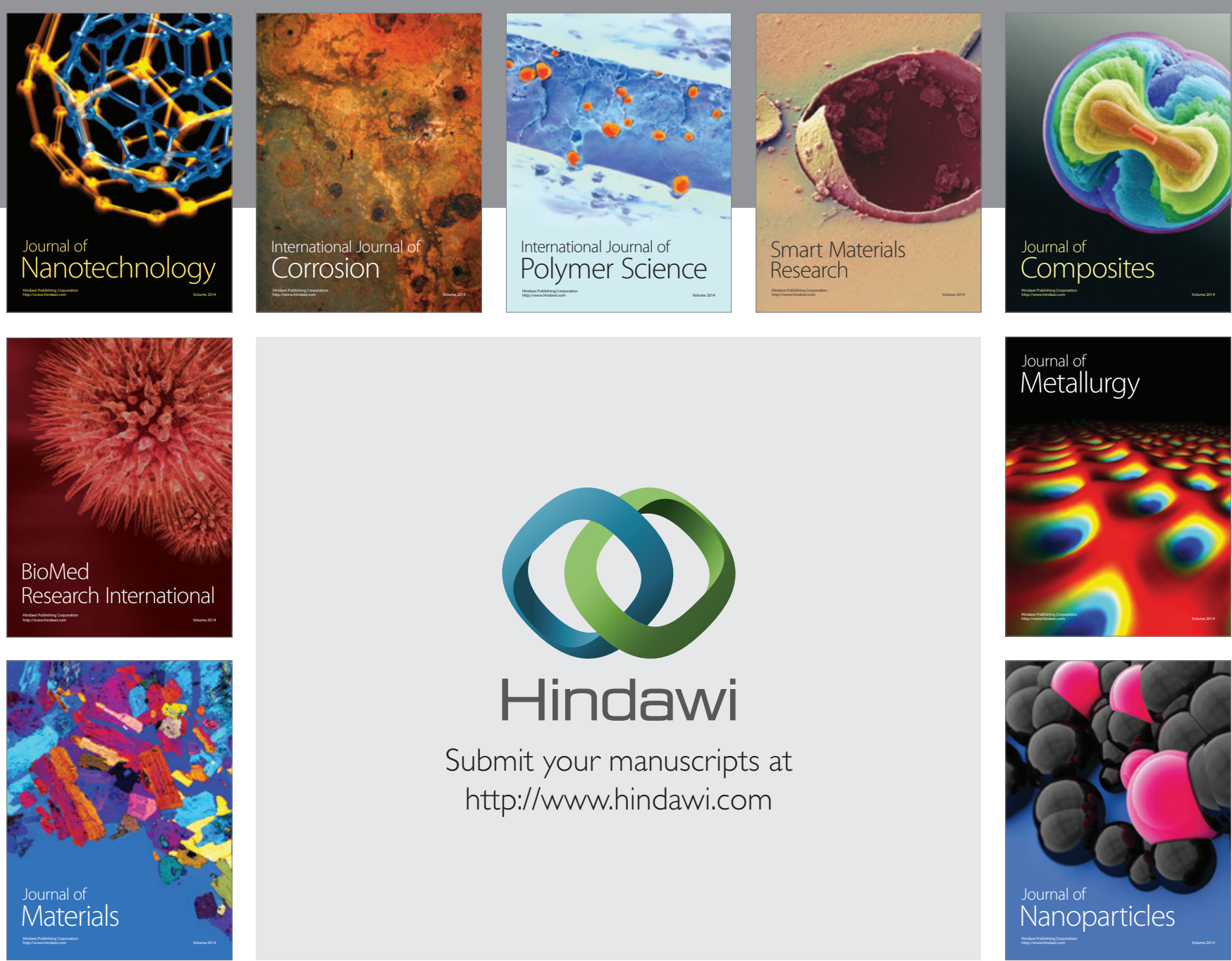

\section{Hindawi}

Submit your manuscripts at

http://www.hindawi.com

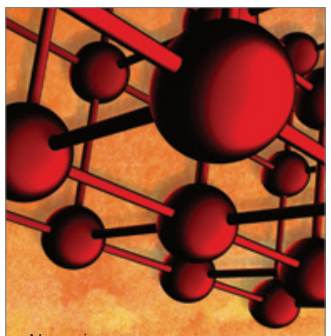

Materials Science and Engineering
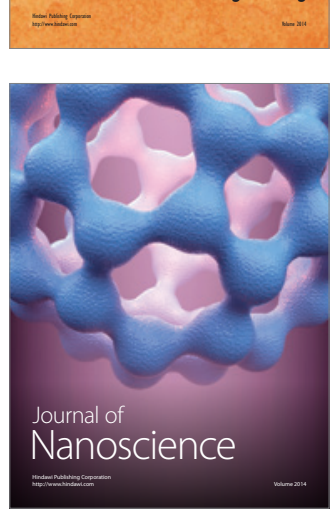
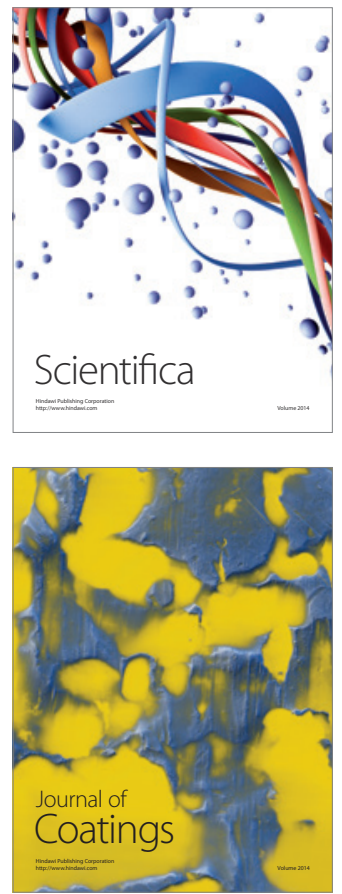
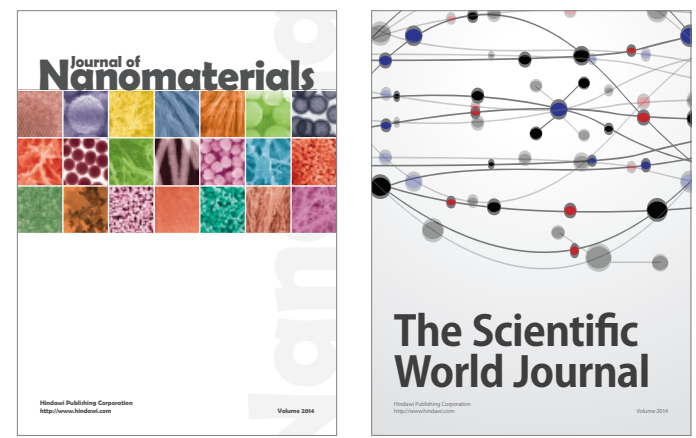

The Scientific World Journal
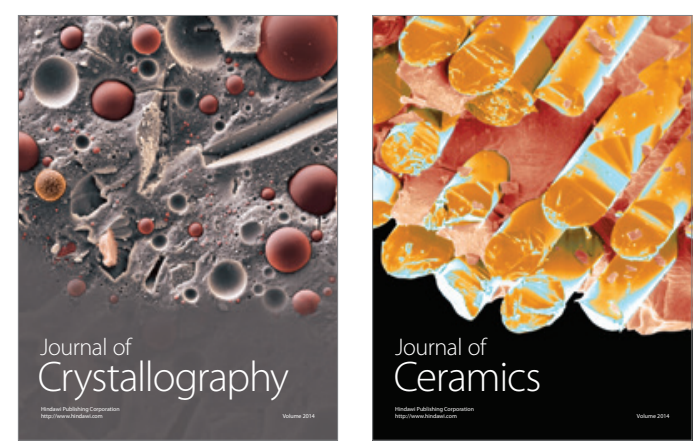
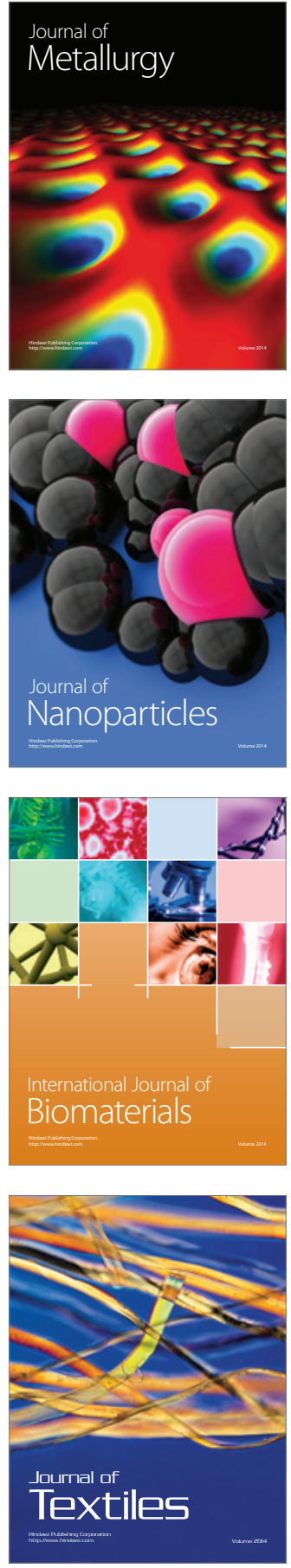